\title{
Cutaneous atypical mycobacteriosis with sporotrichoid clinical presentation caused by automotive accident*
}

\author{
John Verrinder Veasey¹, Nina Angélica Souza Monteiro ${ }^{1}$, Rute Facchini Lellis², Giselle Burlamaqui \\ Klautau ${ }^{3}$
}

DOI: http://dx.doi.org/10.1590/abd1806-4841.20187586

\begin{abstract}
Atypical mycobacterial infections are caused by mycobacteria other than those from the $M$. tuberculosis complex and M. leprae. Its incidence has increased progressively, with considerable increase of scientific publications on the subject. Only $10 \%$ of the cases present with cutaneous infections, most of them related to surgical interventions and aesthetic procedures. We present a case of mycobacteriosis due to automotive accident that presented a diagnostic challenge due to its clinical presentation with lesions of sporotrichoid progression in the lymphatic system.
\end{abstract}

Keywords: Mycobacterium infections, nontuberculous; Nontuberculous mycobacteria; Soft tissue injuries; Sporotrichosis; Traumatology; Wounds and injuries

Mycobacteria are agents classified in two groups according to the risk criteria for infection in humans: a group of strict pathogens, including the agents for tuberculosis and Hansen's disease, and another group of species considered potentially pathogenic known as "nontuberculous mycobacteria" (NTM), "opportunistic mycobacteria" or "occasional pathogens". ${ }^{1-3}$ Cutaneous infections correspond to nearly $10 \%$ of the cases of infections by NTM. ${ }^{4}$ They occur preferentially in immunosuppressed patients, with a reduction in the number of cases associated to AIDS patients and progressive increase in the number of cases associated to immunosuppressant medications. ${ }^{1,3,5,6}$ Immunocompetent patients have also been affected by NTM infection due to an increase in the number of aesthetic and surgical procedures to which they are submitted, favoring inoculation of the bacteria in the subcutaneous tissue. ${ }^{2,5,7}$
Cutaneous clinical manifestations can be localized or disseminated, with or without associated internal involvement. ${ }^{1,4,5}$ Cases with no internal involvement and localized lesions correspond to Buruli ulcer, aquagenic and nonaquagenic lymphocutaneous lesions (or sporotrichoid) and aquagenic and nonaquagenic nonlymphocutaneous lesions. . $^{1,7}$

We present the case of a male, 29-year-old patient born in Guinea-Bissau, living in São Paulo for 7 years, with the complaint of a 4-month history of a lesion on the right foot after an automotive accident. The lesions started as a dome-shaped tumor with a smooth surface on the plantar aspect of the third, fourth and fifth right toes, progressing after weeks with new lesions as verrucous plaques on the dorsum of the same foot, followed by the appearance of an ulcer on the pretibial region (Figure 1). Due to the aspect of the lesions,

\footnotetext{
Received 25 August 2017.

Accepted 31 March 2018.

* Work conducted at the Dermatology Clinic, Hospital da Santa Casa de São Paulo, São Paulo (SP), Brazil.

Financial support: None.

Conflict of interest: None.

Dermatology Clinic, Hospital da Santa Casa de São Paulo, São Paulo (SP), Brazil.

Laboratory of Pathology, Hospital da Santa Casa de São Paulo, São Paulo (SP), Brazil.

Infectious Diseases Clinic, Hospital da Santa Casa de São Paulo, São Paulo (SP), Brazil.

MAILING AdDREsS:

John Verrinder Veasey

E-mail: johnveasey@uol.com.br

C2018 by Anais Brasileiros de Dermatologia
} 
the hypotheses of chromomycosis, sporotrichosis and atypical mycobacteriosis were raised.

Biopsies were taken in series (in a total of 4 ) of the lesions on the soles and dorsum of the foot and the lesion on the leg, and samples were sent for fungal and mycobacterial culture and histopathology. Chest radiography, which showed no abnormalities and tuberculin test, which was positive with a $20 \mathrm{~mm}$ reaction, were also performed. Fungal cultures did not show any growth, and histopathology examination showed a suppurative granulomatous reaction associated to cicatricial fibrosis with Hematoxylin \& eosin stain (Figures 2 and 3). Detection of acid-fast bacilli with the Ziehl-Nielsen method and fungus with Grocott's stain were both negative in
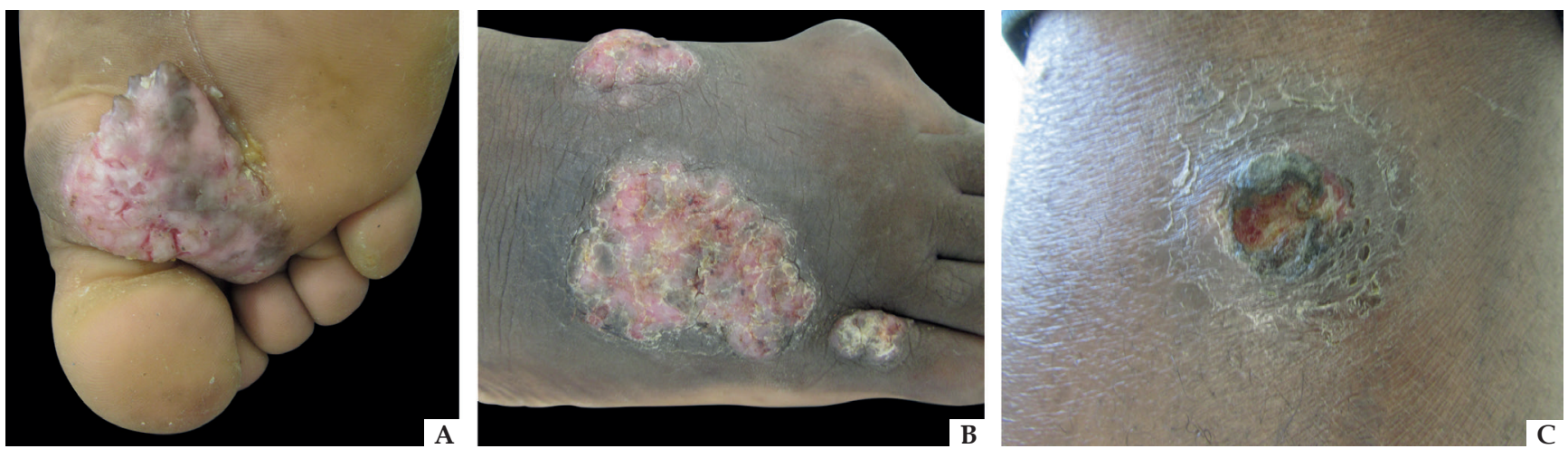

FigURE 1: Initial clinical aspect of the lesions. (A) Initial plantar lesion, (B) lesions on the dorsum of the foot that appeared along the course of the disease and $(\mathrm{C})$ pretibial ulcerated lesion

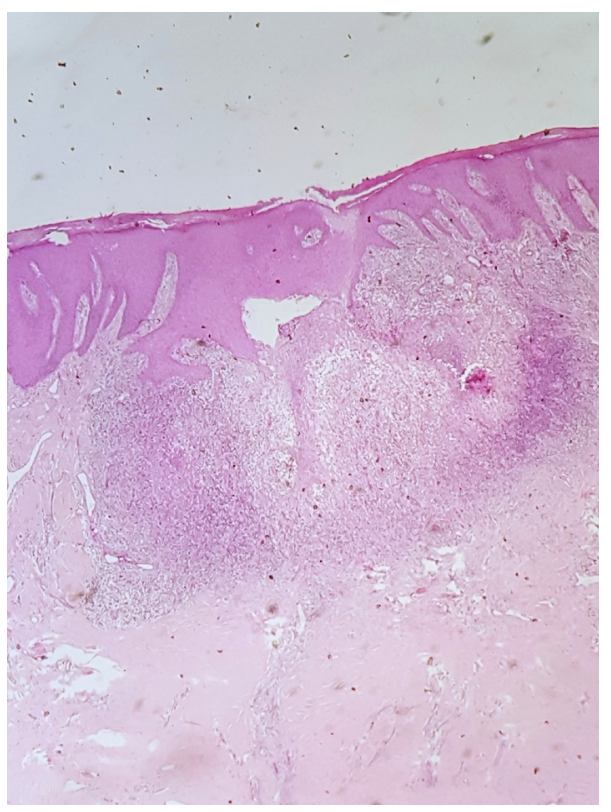

Figure 2:

Suppurative granulomatous nodular process associated to cicatricial fibrosis (Hematoxylin \& eosin, $x 100)$

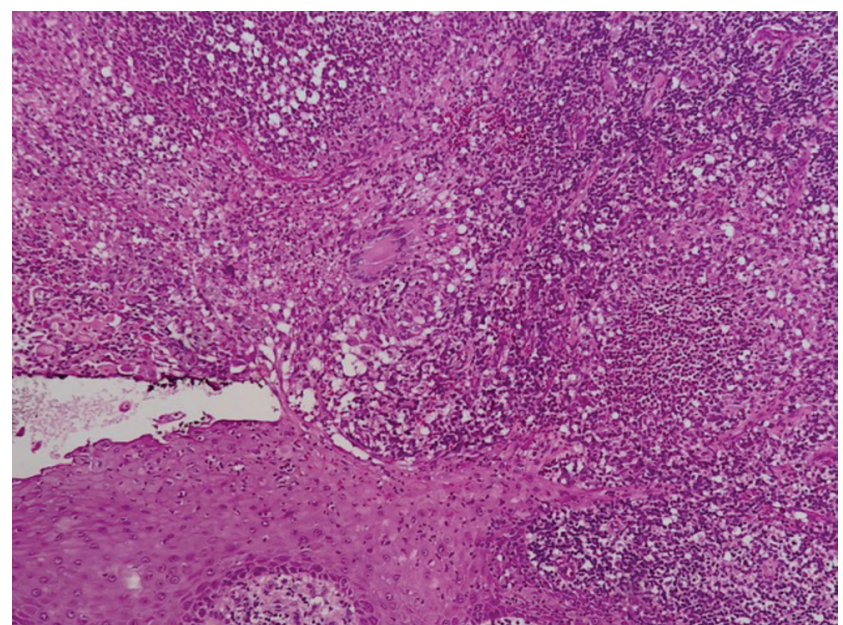

FIGURE 3: Epithelioid granuloma with suppurative center (Hematoxylin \& eosin, x200)
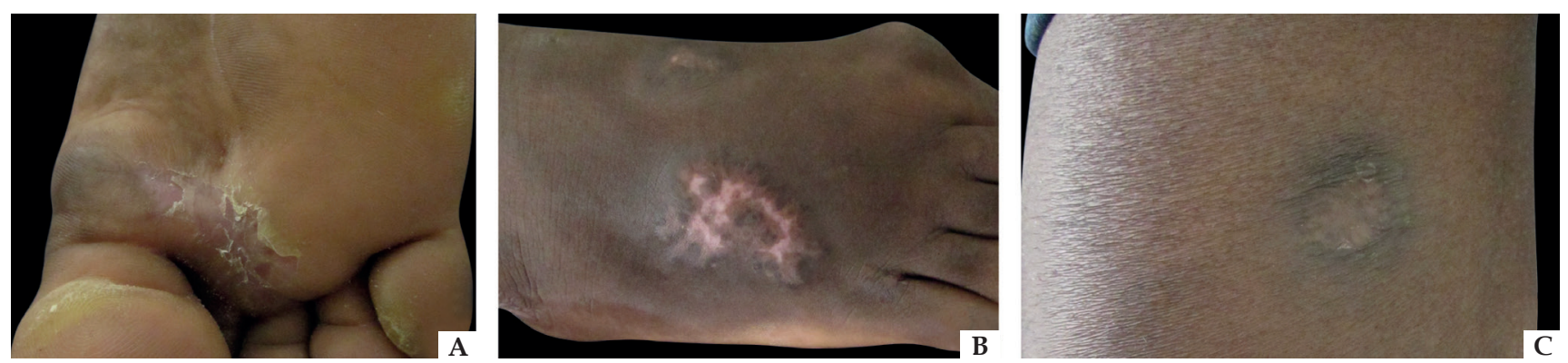

Figure 4: Aspect of the lesions five months after treatment with rifampicin 600mg, isoniazid 300mg, ethambutol 1,200mg, pyridoxine 50mg and clarithromycin 1,000mg (daily doses). (A) Plantar lesion, (B) Dorsum of the foot, and (C) Pretibial lesion 
all samples. There was growth of Mycobacterium sp non-tuberculosis complex in only one sample. With the diagnosis of atypical mycobacteriosis, the patient evolved with marked improvement of the lesions after 5 months of treatment with rifampicin $600 \mathrm{mg}$, isoniazid $300 \mathrm{mg}$, ethambutol 1,200mg, pyridoxine 50mg and clarithromycin 1,000mg (daily doses) (Figure 4).

\section{REFERENCES}

1. Guglielmetti L, Mougari F, Lopes A, Raskine L, Cambau E. Human infections due to nontuberculous mycobacteria: the infectious diseases and clinical microbiology specialists point of view. Future Microbiol. 2015;10:1467-83.

2. Gonzalez-Santiago TM, Drage LA. Nontuberculous Mycobacteria: Skin and Soft Tissue Infections. Dermatol Clin. 2015;33:563-77.

3. Lamb RC, Dawn G. Cutaneous non-tuberculous mycobacterial infections. Int J Dermatol. 2014;53:1197-204.

4. Murback NDN, Higa Júnior MG, Pompílio MA, Cury ESJ, Hans Filho G, Takita LC. Disseminated cutaneous atypical mycobacteriosis by $M$. chelonae after sclerotherapy of varicose veins in a immunocompetent patient: a case report. An Bras Dermatol. 2015;90:S138-42.
Atypical mycobacterial infections are among the main causes of infectious lesions with sporotrichoid distribution in Europe. The patient was seen in Brazil, where sporotrichosis is endemic and was one of the main differential diagnoses. Despite the agent not being identified on histopathology, the cutaneous reaction pattern found is consistent with atypical mycobacterial infection. ${ }^{2,4,5}$ In a review with 13 cases of atypical mycobacterial infections, the agent was only isolated in culture in 7 of them, such as in our case, and the others had a positive PCR. ${ }^{5} \square$

\section{AUTHORS'CONTRIBUTIONS \\ John Verrinder Veasey $\quad$ (iD) ORCID 0000-0002-4256-5734 \\ Approval of the final version of the manuscript, Design and planning of the study, Preparation and writing of the manuscript \\ Nina Angélica Souza Monteiro \\ (iD) ORCID 0000-0003-0856-1489 \\ Preparation and writing of the manuscript, Collecting, analysis and interpretation of data}

How to cite this article: Veasey JV, Monteiro NAS, Lellis RF, Klautau GB. Cutaneous atypical mycobacteriosis with sporotrichoid clinical presentation caused by automotive accident. An Bras Dermatol. 2018;93(5):743-5.

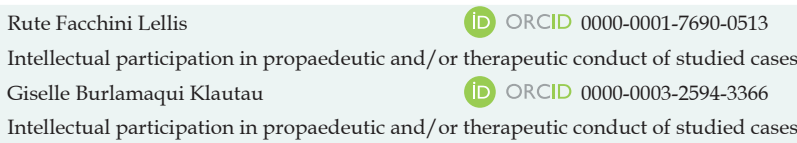

5. Li JJ, Beresford R, Fyfe J, Henderson C. Clinical and histopathological features of cutaneous nontuberculous mycobacterial infection: a review of 13 cases. J Cutan Pathol. 2017;44:433-443.

6. Dodiuk-Gad R, Dyachenko P, Ziv M, Shani-Adir A, Oren Y, Mendelovici S, et al. Nontuberculous mycobacterial infections of the skin: a retrospective study of 25 cases. J Am Acad Dermatol. 2007;57:413-20.

7. Boleira M, Lupi O, Lehman L, Asiedu KB, Kiszewski AE. Buruli ulcer. An Bras Dermatol. 2010;85:281-301. 